\title{
Europa-Studien zwischen Affirmation und EU-Kritik
}

\author{
Timm Beichelt und Christina Ücker*
}

Im Rahmen von Forschung sowie Lehre haben die Europa-Studien in den vergangenen Jahren in starkem Maße an Umfang und Perspektivenreichtum gewonnen. ${ }^{1}$ Die Gründe für diese Entwicklung werden in einem doppelten Veränderungsdruck gesehen. Zum einen haben politische Ereignisse den Bedarf an europawissenschaftlichem Wissen erhöht: die stetige Vertiefung der europäischen Integration seit der Einheitlichen Europäischen Akte (1986), die Erweiterung der Europäischen Union um mehr als ein Dutzend neue Mitglieder seit 1995, die daraus erwachsene sozio-ökonomische und sozio-kulturelle Ausdifferenzierung innerhalb der Europäischen Union sowie nicht zuletzt die krisenhafte Zuspitzung durch die Finanz- und Schuldenkrise. Zum anderen hat die rastlose Umstrukturierung der europäischen Hochschullandschaft vielerorts zu einer Verschiebung von vormals disziplinären oder interdisziplinären zu gegenstandsorientierten Studienangeboten geführt. Dadurch kam es europaweit zu einem Anwachsen an Programmen der Europa-Studien. Vor einigen Jahren konnte für den europäischen Hochschulraum die stattliche Zahl von 305 Programmen mit dem Label der ,European Studies' oder verwandter Bezeichnungen ermittelt werden. ${ }^{2}$

In einem früheren Beitrag in dieser Zeitschrift haben wir versucht herauszuarbeiten, dass ein guter Teil der in diesem Sinne erschaffenen Studienprogramme einem ,orientierungswissenschaftlichen“" Ansatz folgt. ${ }^{3}$ Im vorliegenden Text möchten wir einen Schritt weitergehen und darlegen, in welche Richtungen sich seither das explizit mit ,European Studies` auseinandersetzende Schrifttum erweitert hat und daher eine weiterführende Einordnung des europawissenschaftlichen Ansatzes ermöglicht. Europa-Studien beziehungsweise Europawissenschaften ${ }^{4}$ folgen, so die These, einer dreifachen Agenda. Zunächst haben sie sich aus den orientierungswissenschaftlichen Ansätzen im Sinne europäischer , area studies ‘ entwickelt, die Europa als einen Kultur- und Gesellschaftsraum mit diffusen Grenzen behandeln (1). Europa-Studien basieren in diesem Kontext auf einem dezidiert multidisziplinären Programm mit einem häufig geistes- und/oder geschichtswissenschaftlichen Fokus.

Den ,European area studies' sind die sozialwissenschaftlich ausgerichteten EU-Studien entgegenzusetzen; der wichtigste Gegensatz besteht also in der Trennlinie zwischen ,Europa und ,EU‘. Die EU-Studien haben es sich zur Aufgabe gemacht, den Prozess der europäischen Integration seit 1945 wissenschaftlich, aber auch politisch und gesellschaftspolitisch zu begleiten. Innerhalb der EU-Studien beobachten wir eine weitere Trennlinie. Auf der einen Seite finden sich affirmative Analysen der europäischen Integration (2). Hier geht es zugleich darum, Wissen über den Integrationsprozess zu generieren und einen Integrationsprozess zu

* Prof. Dr. Timm Beichelt, Professur für Europa-Studien, Europa-Universität Viadrina, Frankfurt Oder. Christina Ücker, MA, Europa-Universität Viadrina, Frankfurt Oder.

1 Vergleiche John Keeler: Mapping EU Studies: The Evolution from Boutique to a Boom Field 1960-2001, in: Journal of Common Market Studies 3/2005, S. 551-582.

2 Timm Beichelt/Bożena Chołuj/Gerard Rowe/Hans-Jürgen Wagener/Thekla Lange: Einleitung: Was heißt und zu welchem Ende studiert man Europa-Studien?, in: Timm Beichelt/Bożena Chołuj/Gerard Rowe/Hans-Jürgen Wagener (Hrsg.): Europa-Studien. Eine Einführung, Wiesbaden 2006, S. 7-44, hier S. 13.

3 Timm Beichelt: Europa-Studien in der Politikwissenschaft: Was sollen, was können sie leisten?, in: integration 3/2006, S. 213-218.

4 Beide Begriffe werden im vorliegenden Text synonym verwendet. 
vermitteln, der aus politischen oder gesellschaftlichen Gründen befürwortet wird. Auf der anderen Seite steht (3) die kritische Europawissenschaft, welche die europäische Herrschaftsordnung im Hinblick auf unterschiedliche, real bestehende Defizite infrage stellt und mit den Leistungen anderer, realer oder theoretisch abgeleiteter, Herrschaftsformen vergleicht. Ein wichtiger Unterschied besteht dabei gewissermaßen in der Erzählerperspektive der beteiligten Forscher oder Wissensvermittler. Affirmative EU-Forscher identifizieren sich zumindest implizit mit dem Ziel, durch ihre Wissensgenerierung das europäische Projekt bisweilen tituliert als „europäischer Traum“5 - voranzutreiben. Kritische EU-Forscher sehen davon ab, die europäische Integration im Sinne der politischen Bildung positiv zu bewerten. Sie geraten dabei allerdings in Gefahr, sich gegen einen lange gehegten gesellschaftlichen Konsens zu stellen, der in der Vereinigung Europas ein übergeordnetes Ziel sieht.

\section{Europa-Studien als kulturwissenschaftliche ,area studies ${ }^{6}$}

Das Konzept der zunächst an US-amerikanischen Universitäten entwickelten ,area studies' verfolgt das Ziel, wissenschaftliche Expertise für Regionen bereitzustellen, wobei der Sprachkompetenz ein hoher Stellenwert beigemessen wird. In den 1960er Jahren motivierte der von der britischen Regierung angeforderte Hayter Report dazu, interdisziplinäre Forschungsinstitute für , area studies' mit den anfänglichen Schwerpunkten Asien, Afrika sowie der Sowjetunion einzurichten. Dieser ursprünglich in Großbritannien institutionalisierte, interdisziplinäre, sozialwissenschaftliche Ansatz setzte einen wissensorganisatorischen Wandel in Bewegung. Zum einen bildeten sich regionalspezifisch ausgerichtete Institute heraus und zum anderen orientierte sich die Finanzierung der Geistes- und Sozialwissenschaft zu bestimmten Teilen an realweltlichen Relevanzkriterien. ${ }^{6}$ Mit dem wachsenden Einfluss der Europäischen Wirtschaftsgemeinschaft und dem Beitritt Großbritanniens im Jahr 1973 gewann Europa zudem als Region eine besondere Bedeutung für die , area studies', welche sich von den USA und Großbritannien nach Kontinentaleuropa ausbreiteten. Europa-Studien, die einen Schwerpunkt auf die Entwicklung der Europäischen Union in wirtschaftlicher, rechtlicher sowie gesellschaftlicher Dimension setzen, können aus dieser Perspektive als Sonderform der , area studies' ${ }^{6}$ gesehen werden. ${ }^{7}$

Europa-Studien werden damit auch zum Gegenstand der Debatte über den Sinn der, area studies', die in der anglo-amerikanischen Wissenschaft in den 1980er und 1990er Jahren geführt wurde. Der Vorwurf an regionalwissenschaftliche Ansätze lautete, dass diese zum Senken wissenschaftlicher beziehungsweise disziplinärer Standards neigen würden. Verteidigt wurden sie mit dem Argument, dass gerade die vertiefte Auseinandersetzung mit einzelnen Gegenständen beziehungsweise Gesellschaftsräumen ein fundiertes Verständnis gesellschaftlicher Entwicklungen ermögliche, welches durch indikatorenbasierte Vergleiche nie erreicht werden könne. Vor diesem Hintergrund wurde lange, besonders seitens der Sozialwissenschaften, zurückgewiesen, Europa als eigenständiges Studienobjekt einer Teildisziplin zu berücksichtigen. ${ }^{8}$ Andere Autoren argumentierten dagegen, Europa-Studien seien nicht im Sinne einer disziplinären Spezialisierung zu verstehen, sondern als „,complex

5 Vergleiche Jeremy Rifkin: Der europäische Traum, Frankfurt Main 2004.

6 Vergleiche Sir William Hayter: The Hayter Report and after, in: Oxford Review of Education 2/1975, S. 169-172, hier S. 172

7 Siehe Philomena Murray: European Studies - Looking to the Future, in: Australian and New Zealand Journal of European Studies 1/2009, S. 1-13.

8 Helen Wallace: Studying contemporary Europe, in: The British Journal of Politics \& International Relations 1/2000, S. 95-113, hier S. 97. 
interwoven with ideas about modernity, the West, Christendom, democracy, and civilization itself". ${ }^{9}$ In diesem Kontext erfolgt die Charakterisierung von Europa-Studien als einer Orientierungswissenschaft. Europa-Studien leisten demnach eine Orientierungsfunktion, die sich „,in Form von Argumenten, Entwürfen, Konstruktionen“ zu Fragen moderner Kulturen widerspiegelt. ${ }^{10}$

In diesem Sinne folgen Europa-Studien der kulturwissenschaftlichen Tradition, wie sie in den 1960er Jahren von dem Literaturwissenschaftler Raymond Williams, dem Soziologen Stuart Hall sowie dem Historiker Edward Thompson angeregt wurde. Neben der Abkehr vom bürgerlichen Kulturbegriff und der Hinwendung zur Populärkultur wurde im Zuge des ,cultural turn' ein weiter Kulturbegriff vertreten, dessen Ausprägungsformen die Kulturwissenschaften zu ergründen versuchen. ${ }^{11}$ So versteht Williams Kultur als „whole way of life", woraus sich erklärt, weshalb der Kulturbegriff hier nicht nur bildliche und schriftliche Artefakte, sondern auch gesellschaftliche, wirtschaftliche sowie politische Praktiken umfasst. ${ }^{12}$ Einen weiteren Grund für die verstärkte Berücksichtigung von Kultur bildet die Einsicht, dass viele soziale Phänomene der Gegenwart kaum mit dem Instrumentarium disziplinär organisierter Forschung untersucht werden können. Infolgedessen „kommt die ,Kultur' als ein Bestandteil des Explanans zur Geltung“" kulturelle Faktoren werden zur Erklärung gesellschaftlicher, politischer oder wirtschaftlicher Phänomene herangezogen. Europa-Studien in den Kulturwissenschaften zu verorten heißt demnach, einen Pluralismus an Ansätzen und Wissensbeständen einem orientierungswissenschaftlichen Paradigma zuzuordnen, um einen multidisziplinären Raum zuzulassen, ,an dem sich moderne Gesellschaften ein Wissen von sich selbst in Wissenschaftsform verschaffen". ${ }^{13}$

Vor diesem Hintergrund hat sich in den vergangenen zwei Jahrzehnten eine Europawissenschaft etabliert, welche die fortschreitende Integration der europäischen Wirtschaft und Politik auf ihre kulturellen und historischen Fundamente abklopft. Zu nennen wären der Berliner Historiker Hartmut Kaelble, der in Erfurt und Freiburg tätige Soziologie Hans Joas oder der kürzlich verstorbene Geschichtssoziologe Willfried Spohn. Wenngleich es verfehlt wäre, von einer Schule zu sprechen, scheinen diese und weitere Wissenschaftler in der Überzeugung vereint, der Charakter - und möglicherweise der Erfolg oder Misserfolg - der europäischen Integration stünde nicht nur in engem Zusammenhang mit kulturellen und historischen Wurzeln, sondern auch mit deren Interpretation durch Individuen, Öffentlichkeiten und Gesellschaften. So beginnen Hans Joas und Klaus Wiegandt in einem Herausgeberwerk über „die kulturellen Werte Europas“ mit einem Ausschnitt aus einem Roman von Joseph Roth, in welchem ein Dialog über Werte der Religion, der Gesittung und der Kunst als „Idee Europa“ bezeichnet werden. ${ }^{14}$ Im Mittelpunkt steht damit nicht die Substanz bestimmter Traditionen - infrage kommen bei Joas und Wiegandt etwa das jüdisch-christliche, das grie-

9 Craig Calhoun: European Studies: always already there and still in formation, in: Comparative European Politics $1 / 2003$, S. 5-20, hier S. 6

10 Jürgen Mittelstraß: Glanz und Elend der Geisteswissenschaften. Oldenburger Universitätsreden Nr. 27, Oldenburg 1989, S. 5-33, hier S. 18.

11 Siehe dazu Dariusz Aleksandrowicz/Christa Ebert/Heinz Dieter Kittsteiner u.a.: Vorwort: Was sind Kulturwissenschaften? 13 Antworten, in: Heinz Dieter Kittsteiner (Hrsg.): Was sind Kulturwissenschaften? 13 Antworten, München 2004, S. 7-24, hier S. 3.

12 Vergleiche Raymond Williams: Culture and Society, Harmondsworth 1963, S. 16.

13 Wolfgang Frühwald/Hans Robert Jauß/Reinhart Koselleck/Jürgen Mittelstraß/Burkhart Steinwachs: Geisteswissenschaften heute. Eine Denkschrift, Frankfurt Main 1991, S. 43.

14 Hans Joas/Klaus Wiegandt: Einleitung, in: Hans Joas/Klaus Wiegandt (Hrsg.): Die kulturellen Werte Europas. Frankfurt Main, 2005, S. 11-12. 
chisch-römische, das rationalistische und das aufklärerische Erbe ${ }^{15}$-, sondern deren Spiegelung durch Interpreten und Interpreten der Interpreten. Es handelt sich um eine Herangehensweise, die aus der kulturwissenschaftlichen Kommunikationsforschung hinlänglich bekannt ist. ${ }^{16}$

Darüber hinaus stellt die Auseinandersetzung mit Grundlagen und Grenzen des Erkenntnisvermögens ein typisches Diskussionsfeld der , area studies' dar. Man denke an die umfassende Debatte, die innerhalb der deutschen Osteuropaforschung regelmäßig aufkeimt. ${ }^{17}$ In den gegenwärtigen kulturwissenschaftlichen Europa-Studien findet die Selbstvergewisserung allerdings vor dem Hintergrund einer stetig wachsenden Aufmerksamkeit sowie Ausstattung statt. Es fehlt der alarmistische Unterton, welcher innerhalb der Debatte der , area studies' häufig mitschwingt. ${ }^{18}$ Vielmehr wird Europa in verschiedenen Disziplinen als globales Vorbildmodell konstruiert. Gefragt wird etwa, warum gerade in Europa der ,Sonderweg ${ }^{6}$ von Industrialisierung, Aufklärung und Kapitalismus beschritten werden konnte. ${ }^{19}$ Im Grunde verweist dies auf eine andauernde Diskussion, die zu Beginn des 20. Jahrhunderts von Max Weber angestoßen wurde und die sich nicht auf historische Entwicklungspfade beschränkt. ${ }^{20}$ Stattdessen wird eine Überlegenheit des gegenwärtigen europäischen Integrationskurses behauptet, wobei die zu zitierenden Arbeiten aus der Zeit vor Beginn der europäischen Schulden- und Finanzkrise stammen. In diesen Kontext gehört die oben bereits erwähnte These Jeremy Rifkins vom „europäischen Traum“.21 In eine ähnliche Richtung argumentiert Steven Hill, welcher Europa und der Europäischen Union eine globale politische Überlegenheit in vielen Politikfeldern und hinsichtlich der inneren Herrschaftsorganisation attestiert. ${ }^{22}$ Der auf Vielfalt und historisch gewachsenen Kompromissen gegründete europäische Gesellschafts- und Herrschaftsraum wird dabei in einen expliziten Vergleich zu anderen Regionen gesetzt. ${ }^{23}$

Insofern läuft aus unserer Sicht der Vorwurf in die Irre, Europa-Studien seien vorrangig euro-zentristisch geprägt und daher blind für andere Kulturräume. ${ }^{24}$ Stattdessen gewinnen Europa-Studien in ihrem eigenen Selbstverständnis durch den Vergleich:

15 Im genannten Herausgeberwerk finden sich einschlägige Texte von Wolfgang Huber (,jüdisch-christliche Tradition“), Christian Meier (,griechisch-römische Tradition“), Wolfgang Schluchter (,Rationalität - das Spezifikum Europas?“) und Reinhart Koselleck („Über den Stellenwert der Aufklärung in der deutschen Geschichte“). Vgl. Joas/Wiegandt: Die kulturellen Werte Europas, 2005.

16 Angelika Linke: Kommunikation, Kultur und Vergesellschaftung. Überlegungen zu einer Kulturgeschichte der Kommunikation, in: Ludwig M. Eichinger/Heidrun Kämper (Hrsg.): Sprache - Kognition - Kultur. Sprache zwischen mentaler Struktur und kultureller Prägung, Berlin/New York 2008, S. 24-50.

17 Stefan Creuzberger/Ingo Mannteufel/Alexander Steininger/Jutta Unser (Hrsg.): Wohin steuert die Osteuropaforschung? Eine Diskussion, Köln 2000.

18 Ein anderes Beispiel bilden die ,German Studies', welche sich in den USA in einem Schrumpfungsprozess befinden, vergleiche dazu Anna Polonyi: German, no thanks, in: Tageszeitung, 15.2.2012.

19 Michael Mitterauer: Warum Europa? Mittelalterliche Grundlagen eines Sonderwegs, München 2000.

20 Max Weber: Die protestantische Ethik und der,Geist‘ des Kapitalismus, Original 1904, München 2010. Weber hatte seinen gesellschaftshistorischen Zugriff bekanntlich ebenfalls als „kulturwissenschaftliche“ Herangehensweise charakterisiert. Vgl. Max Weber: Die ,Objektivität‘ sozialwissenschaftlicher und sozialpolitischer Erkenntnis, in: Max Weber (Hrsg.): Gesammelte Aufsätze zur Wissenschaftslehre, Original 1904, Tübingen 1985, S. 146-214.

21 Rifkin: Der europäische Traum, 2004.

22 Vgl. Steven Hill: Europe's Promise. Why the European Way is the Best Hope in an Insecure Age, Berkeley 2010.

23 Siehe auch Robert Kagan: Power and Weakness. Why the United States and Europe see the world differently, in: Policy Review 113/2002, S. 3-28.

24 So Dipesh Chakrabarty: Europa provinzialisieren. Postkolonialität und die Kritik der Geschichte, in: Sebastian Conrad/Shalini Randeria (Hrsg.): Jenseits des Eurozentrismus, Frankfurt Main 2002, S. 283-312, hier S. 283. 
„Mit dem Zusammenbruch der Ideologien haben die Weltreligionen und ihre kulturellen Kontexte erneut an Bedeutung gewonnen [...] In diesen kulturellen Kämpfen hat Europa historische Erfahrungen. Jede Gruppierung, jede Nation, hat sich schon einmal aus der Perspektive der Verlierer betrachten dürfen. Kurzfristig schärft das das Ressentiment - langfristig schärft es den Verstand.“25

Somit kennzeichnet den Mainstream der kulturwissenschaftlichen Europa-Studien eine in globale Kontexte eingebettete Erörterung der Eigenarten des ,European way'. Sie basiert darauf, die vielfältigen und mitunter divergierenden europäischen Gesellschaften und Kulturen zu erfassen. Charakteristisch ist, dass in vielen Publikationen nicht nur die einenden und trennenden gesellschaftlichen Merkmale der Europäer herausgearbeitet werden, sondern damit zugleich die Methode des historischen Vergleichs eingeführt wird. ${ }^{26}$ Einen stärker soziologischen Zugriff auf die geschichtliche Entwicklung Europas pflegen Shmuel Eisenstadt und Willfried Spohn. Beide gehen davon aus, Europa als Raum der multiplen Modernität aufzufassen. ${ }^{27}$ Sie ordnen dem westeuropäischen Raum folgende Begriffe zu: „ökonomische Entwicklung, innere Staatsbildung, nationale Homogenisierung und mangelnde Staatsbildung“. ${ }^{28}$ Mittel-, Südost- und Osteuropa werden zu den genannten Begriffen in Gegensatz gebracht, wobei Mitteleuropa als Region betrachtet wird, welche in jüngerer Zeit den Pfad der ,Modernität‘ eingeschlagen habe. Dem außereuropäischen Vergleich gesellt sich daher in kulturwissenschaftlichen Europa-Studien auch eine innereuropäische Vergleichsperspektive, welche freilich nicht nur im Paradigma der soziologischen Modernisierung stattfindet. ${ }^{29}$

Insgesamt zeigt sich, dass die kulturwissenschaftliche Wendung der frühen 1990er Jahre die Europa-Studien stark inspiriert hat. Es kann zwar nicht von der Existenz einer europawissenschaftlichen Transdisziplin mit ,integrierenden und die alten Disziplinen übertreffenden Deutungsanspruch “30 ausgegangen werden. Allerdings lässt sich eine allmähliche geistes- und sozialwissenschaftliche Europäisierung in dem Sinne beobachten, dass der noch vor wenigen Jahren oft bedauerte „,methodologische Nationalismus“31 erkannt wurde und vor der Überwindung steht. Viele Forscher nehmen die „,nationale Begrenzung des Forschungshorizonts“332 als Problem wahr und weichen auf transnationale Konstruktionen, wie

25 Heinz Dieter Kittsteiner: Europa. Anmerkungen zur Genese eines rastlosen Kontinents, in: Timm Beichelt/ Bożena Chołuj/Gerard Rowe/Hans-Jürgen Wagener (Hrsg.): Europa-Studien. Eine Einführung, Wiesbaden 2006, S. 49-64, hier S. 60.

26 Stellvertretend genannt sei Hartmut Kaelble: Eine europäische Gesellschaft?, in: Gunnar Folke Schuppert/Ingolf Pernice/Ulrich Haltern (Hrsg.): Europawissenschaft, Baden-Baden 2005, S. 299-330; Hartmut Kaelble: Der historische Vergleich. Eine Einführung zum 19. und 20. Jahrhundert, Frankfurt Main 1999.

27 Shmuel N. Eisenstadt: European civilization in a comparative perspective. A study in the relations between culture and social structure, Oslo 1987; Shmuel N. Eisenstadt: Die Vielfalt der Moderne, Weilerswist 2000; Willfried Spohn: Interdisziplinäre Europa-Studien: der Ansatz der multiplen Moderne, in: Timm Beichelt/ Bożena Chołuj/Gerard Rowe/Hans-Jürgen Wagener (Hrsg.): Europa-Studien. Eine Einführung, Wiesbaden 2006, S. 435-452.

28 Spohn: Interdisziplinäre Europa-Studien, 2006, S. 439.

29 Siehe etwa Helmut Thome: Wertewandel in Europa aus der Sicht der empirischen Sozialforschung, in: Hans Joas/Klaus Wiegandt (Hrsg.): Die kulturellen Werte Europas, Frankfurt Main 2005, S. 386-443.

30 Friedrich Jaeger/Hans Joas: Europa im Spiegel der Kulturwissenschaften, Baden-Baden 2008, S. 12.

31 Vgl. zum Beispiel Anthony Giddens: The Nation State and Violence, Cambridge 1985, S. 172.

32 Beate Kohler-Koch: Die Europäisierung der politikwissenschaftlichen Europaforschung, in: Friedrich Jaeger/ Hans Joas (Hrsg.): Europa im Spiegel der Kulturwissenschaften, Baden-Baden 2008, S. 17-43, hier S. 18. 
den europäischen Kosmopolitismus ${ }^{33}$ oder die europäische Identität, ${ }^{34}$ aus: „Europa wird als ein Geschehensraum sichtbar". ${ }^{35}$ Damit zeigen kulturwissenschaftliche Europa-Studien einen Weg aus vormals national orientierten Wissenschaftskulturen.

In der Binnenorganisation multidisziplinärer ,area studies' stellt sich zudem die Frage, wie das Zusammenspiel der einzelnen Disziplinen organisiert werden soll. Interessanterweise lässt sich feststellen, dass sich in der gewöhnlich stark selbstreflexiv ausgerichteten Literatur nur sehr wenige Aussagen finden lassen, wie Interdisziplinarität konkret praktiziert wird oder praktiziert werden sollte. Als „weitgehend eigenständige ,soziale Orte“ der Erkenntnis- und Ausbildungsproduktion“"36 - dies wäre ein Kennzeichen von , area studies ‘ werden Europa-Studien selten wahrgenommen. Sofern dies doch der Fall ist, wird den beteiligten Disziplinen eine hohe Autonomie eingeräumt. Die in Bristol lehrende Michelle Cini hält Ansätze, die von einer Verschmelzung der Disziplinen ausgehen, für kaum realisierbar; in ihrer Terminologie bevorzugt sie den Begriff der Multidisziplinarität, welcher - im Gegensatz zur Interdisziplinarität - disziplinäre Grenzen akzeptiert. ${ }^{37}$ Dies ermöglicht, verschiedene disziplinäre Ansichten zur Problemlösung heranzuziehen, ohne eine Entwicklung theoretischer Synthesen anzustreben. ${ }^{38}$ Vor diesem Hintergrund werden Europa-Studien zu einem instruktiven Forschungsfeld, in dem verschiedene disziplinäre Denkweisen den Gegenstand der kulturellen, gesellschaftlichen und politischen Entwicklung in Europa zu erhellen verhelfen. ${ }^{39}$

\section{EU-Studien als sozialwissenschaftliche Integrationsforschung}

Die EU-Studien als ein Teilgebiet der Europa-Studien verfügen auf der einen Seite über gemeinsame Wurzeln mit den kulturwissenschaftlichen, area studies'. Auf der anderen Seite haben sie sich jedoch in den letzten Jahrzehnten deutlich von diesen entfernt. Die Gemeinsamkeit besteht darin, dass sich die frühe Europawissenschaft aus einem überschaubaren, aber multidisziplinären Wissenschaftlerkreis zusammensetzte. Für den deutschen Raum wäre hierfür beispielsweise der 1969 gegründete Arbeitskreis Europäische Integration zu nennen, in dem sich Rechts-, Wirtschafts-, Politik- und Geschichtswissenschaftler organisieren. Im Zuge des Ausdifferenzierungsprozesses innerhalb der Geisteswissenschaften während der 1970er und 1980er Jahre ging aus der breiter angelegten Europawissenschaft eine For-

33 Vgl. Ulrich Beck: Globale Ungleichheit und Menschenrechte: Eine kosmopolitische Perspektive, in: Gerald Beck/Cordula Kropp (Hrsg.): Gesellschaft innovativ, Wiesbaden 2012, S. 273-296.

34 Peter Wagner: Hat Europa eine kulturelle Identität?, in: Hans Joas/Klaus Wiegandt (Hrsg.): Die kulturellen Werte Europas, Frankfurt Main 2005, S. 494-512.

35 Jaeger/Joas: Europa im Spiegel der Kulturwissenschaften, 2008, S. 8.

36 Jürgen Klüver: Wissenschaft und Forschung als Gegenstand der Hochschulforschung, in: Dietrich Goldschmidt/ Ulrich Teichler/Wolff-Dietrich Weber (Hrsg.): Forschungsgegenstand Hochschule. Überblick und Trendbericht, Frankfurt Main 1984, S. 29-51, hier S. 50.

37 Siehe Michelle Cini: The 'State of the Art' in EU Sudies: From Politics to Interdisciplinary (and Back Again?), in: Politics 1/2006, S. 38-46.

38 Laut Ross zeichnen sich disziplinenübergreifende Ansätze durch einen inter- sowie multidisziplinären Mittelweg aus, wobei verschiedene Konzepte, Theorien und Ideen zur Problemlösung herangezogen werden. Ausführlich dazu Fiona Ross: Degrees of Disciplinarity in Comparative Politics: Interdisciplinarity, Multidisciplinarity and Borrowing, in: European Political Science 8/2009, S. 26-36, hier S. 29.

39 Hierzu Michael Moran: Interdisciplinarity and political science, in: Politics 2/2006, S. 73-83. 
schungsrichtung hervor, die sich immer stärker auf den Prozess der europäischen Integration ausgerichtet hat. ${ }^{40}$

Vertreter der EU-Studien halten stärker an disziplinären methodischen Standards fest; häufig im Sinne einer Verengung auf die Internationalen Beziehungen, die Vergleichende Politikwissenschaft oder noch spezifischere theoretische Zugänge. ${ }^{41}$ Hierbei wird ein wesentlicher Schwerpunkt auf institutionelle sowie politische Dimensionen der Integration gelegt. ${ }^{42}$ Im Mittelpunkt der EU-Studien stehen daher Fragen nach Umfang, Merkmalen und Entwicklung der Integration, ${ }^{43}$ was sie von kulturwissenschaftlichen Europa-Studien deutlich unterscheidet, selbst wenn die Begriffe nicht einheitlich und häufig unscharf verwendet werden: „the division between ,European“ and ,EU“ studies is often fuzzy“ ${ }^{44}$ Dennoch ist zweifellos festzuhalten, dass sich EU-Studien im Laufe der Jahre als ein Zweig der sozialwissenschaftlichen Europawissenschaft etabliert haben. ${ }^{45}$ EU-Studien haben sich insofern zu einer quasi-disziplinären Angelegenheit entwickelt, dass ein einigermaßen fest umrissenes Forschungsfeld entstanden ist, in dem sich eine größere Zahl von Forschern auf gemeinsame paradigmatische Grundfeste (im Sinne von Thomas Kuhn) ${ }^{46}$ berufen. Der Unterschied zu einer ,echten' Disziplin besteht darin, als der Gegenstand geografisch definiert ist (im Unterschied zu einem systemisch-phänomenischen Gegenstand wie etwa der ,Gesellschaft ${ }^{\star}$ für die Soziologie) und dass für dessen Erforschung Versatzstücke aus bestehenden Disziplinen, insbesondere der Politik- und Rechtswissenschaft, herangezogen werden.

In der Bundesrepublik lieferte die Deutsche Forschungsgemeinschaft mit dem von 1996 bis 2003 durchgeführten Forschungsprogramm „Regieren in der Europäischen Union“ ”einen Impuls zur akademischen Auseinandersetzung mit Grundsatzfragen zum Funktionieren der Europäischen Union und deren Auswirkungen auf die Nationalstaaten. ${ }^{47}$ Neben der Förderung des disziplinären Austauschs wurde damit unter anderem einer in den 1980er Jahren von Wolfgang Wessels geäußerten wissenschaftspolitischen Kritik begegnet. Demzufolge entwickelte sich der damalige europawissenschaftliche ,acquis academique‘ lediglich im Rahmen neuer empirischer Erkenntnisse. ${ }^{48}$ Eine Absicht des Forschungsprogramms lag in der Bildung von Projekten, welche methodische sowie theoretische Ansätze aus Subdiszi-

40 Peter Bender: Das Ende des ideologischen Zeitalters. Die Europäisierung Europas, Berlin 1981; Werner Weidenfeld/Wolfgang Wessels: Jahrbuch der Europäischen Integration, Bonn 1981; Walter Laqueur: A Continent Astray: Europe 1970-1978, New York 1979.

41 Ausführlich dazu Helen Wallace: Studying contemporary Europe, in: The British Journal of Politics \& International Relations 1/2000, S. 95-113. Rosamond entwickelte im Rahmen dieser Diskussion das „,mainstreaming“ beziehungsweise das ,pluralistic model“, um die unterschiedlichen Standpunkte idealtypisch zu veranschaulichen. Vgl. Ben Rosamond: The political sciences of European integration: disciplinary history and EU studies, in Knud Erik Jørgensen/Mark Pollack/Ben Rosamond (Hrsg.): Handbook of European Union politics, London 2007, S. 7-30, hier S. 14-15.

42 Vergleiche Monika Eigmüller/Steffen Mau: Gesellschaftstheorie und Europapolitik: sozialwissenschaftliche Ansätze zur Europaforschung, Wiesbaden 2010, S. 15.

43 Vergleiche Chris Rumford: Introduction: The Stuff of European Studies, in: Chris Rumford (Hrsg.): The Sage Handbook of European Studies, London 2009, S. 1-14, hier S. 2.

44 Alex Warleigh-Lack: Learning from Europe? EU Studies and the Re-thinking of 'International Relations', in: European Journal of International Relations 1/2006, S. 31-51, hier S. 44-45 und auch S. 34.

45 Siehe Ben Rosamond: European Integration and the Social Science of EU Studies: The Disciplinary Politics of a Subfield, in: International Affairs 2/2007, S. 231-252.

46 Vergleiche Thomas Kuhn: Die Struktur wissenschaftlicher Revolutionen, Frankfurt Main 1976.

47 Ausführliche Informationen dazu sind in der GEPRIS-Datenbank der DFG abrufbar unter:http://gepris.dfg.de/ gepris/OCTOPUS/;jsessionid=A2D23F3F34885631E823EBF01EB632BF?module=gepris\&task=showDetail \& context=projekt\&id=5467626 (letzter Zugriff: 15.12 . 2011).

48 Wolfgang Wessels: Bilanz der wissenschaftlichen Debatte zur Europapolitik 1980-90, in: Werner Weidenfeld Wolfgang Wessels (Hrsg.): Jahrbuch der Europäischen Integration 1980-1990 - eine Bilanz, Bonn 1990, S. 21-31. 
plinen zusammenführen sollten. Die Forschungsprojekte blieben jedoch disziplinär konzipiert. ${ }^{49}$

Die Entwicklung von der integrierten Europawissenschaft zur spezialisierten Integrationswissenschaft lässt sich an Hand einiger Texte nachvollziehen. Im Kontext der schwerwiegenden Folgen zweier Weltkriege in Europa prägte besonders die Frage nach der Vermeidung von Krieg die ersten integrationswissenschaftlichen Theorien. An Immanuel Kants Ausführungen „Zum Ewigen Frieden“ anknüpfend, betrachten Vertreter des Funktionalismus überstaatliche Kooperation als Schlüssel zum Frieden in Europa. ${ }^{50}$ David Mitrany erörtert in seinem Hauptwerk „A Working Peace System“, dass vor dem Hintergrund des transnationalen Charakters vieler staatlicher Herausforderungen, überstaatliche, an Sachlogik geknüpfte, funktionale Kooperation der einzige Weg zu Friedenssicherung sei. ${ }^{51}$ Von dieser Argumentation ausgehend entwickelten sich die konkurrierenden Interpretationsangebote des Neofunktionalismus sowie des Neorealismus, ${ }^{52}$ welche die Ansätze des Supranationalismus und des liberalen Intergouvernementalismus beeinflussten. ${ }^{53} \mathrm{Im}$ zuletzt zitierten Text bezeichnet Andrew Moravcsik die Europäische Union als ein ,internationales Regime für Policy-Koordination“. 54 Dieses Zitat bringt auf den Punkt, dass sich die Herangehensweise der Integrationsforschung von der multidisziplinären Auseinandersetzung mit einem Phänomen sui generis zu einem quasi-disziplinären Vorgehen entwickelt hat: der Regimebegriff entstammt sowohl der binnenstaatlichen Herrschaftslehre als auch der Lehre der Internationalen Beziehungen, der Begriff der Policy-Koordination verweist auf Wurzeln in der Politikfeldforschung sowie der Entscheidungstheorie. Damit hat sich die Europäische Union - wie es lange Zeit gefordert wurde ${ }^{55}$ - als Katalysator zur Verknüpfung innerhalb der Politikwissenschaft angesiedelter Subdisziplinen erwiesen.

Als wichtigster Ausgangspunkt erwies sich dabei die politikwissenschaftliche Subdisziplin der Internationalen Beziehungen. ${ }^{56}$ Die Zunahme politischer Prozesse auf europäischer Ebene wurde lange Zeit als Spezialfall weltpolitischer Integrationskonzepte gesehen, ${ }^{57}$ deren Besonderheit in einem außerordentlich hohen Regelungsniveau bestand. ${ }^{58}$ Die davon stimulierte „politikorientierte Begleitforschung“59 flankiert Kompetenzgewinne der Europäischen Union und ergründet damit die unterschiedlichen Dimensionen der Integration.

49 Kohler-Koch: Die Europäisierung der politikwissenschaftlichen Europaforschung, 2008, S. 23.

50 Siehe Immanuel Kant: Zum Ewigen Frieden, herausgegeben von Rudolf Malter, Stuttgart 2005

51 Vergleiche David Mitrany: A Working Peace System, Chicago 1966.

52 Siehe Ernst Haas: The uniting of Europe: political, social and economic forces, 1950-1957, Stanford 1958; Kenneth Waltz: Theory of International Politics, New York 1979, S. 127; Stanley Hoffmann: Obstinate or Obsolete? France, European Integration and the Fate of the Nation State, in: The European Sisyphus: Essays on Europe 1964-1994, Boulder 1995, S. 71-106.

53 Dazu Andreas Nölke: Supranationalismus, in: Hans-Jürgen Bieling/Marika Lerch (Hrsg.): Theorien der europäischen Integration, Wiesbaden 2005, S. 145-168; Andrew Moravcsik: Preferences and Power in the European Community. A Liberal Intergovernmentalist Approach, in: Journal of Common Market Studies 4/1993, S. 472-524.

54 Moravcsik: Preferences and Power, 1993, S. 480.

55 Siehe John Peterson: The choice for EU theorists: Establishing a common framework for analysis, in: European Journal of Political Research 3/2001, S. 298-318, hier S. 313. „EU scholars need to stop fighting phoney theoretical wars, make choices about what they want to explain, and make the European Union a touchstone in the more general effort in political science to synthesise insights from comparative politics, international relations and public policy“.

56 Vergleiche Andrew Hurrell/Anon Menon: Politics like Any Other? Comparative Politics, International Relations and the Study of the EU, in: West European Politics 2/1996, S. 386-402.

57 Siehe Kees van der Pijl: Vordenker der Weltpolitik, Opladen 1996.

58 Giandomenico Majone: Regulating Europe, London 1996.

59 Kohler-Koch: Die Europäisierung der politikwissenschaftlichen Europaforschung, 2008, S. 22. 
Eine Spielart besteht darin, die Europäische Union als politisches System zu konzipieren und zu untersuchen. ${ }^{60}$ Neben Entscheidungsprozessen, institutionellen Strukturen oder funktionalen Fragen gewannen hierdurch auch Aspekte wie Systemleistung oder Legitimitätsfragen an Bedeutung, womit ein in den Internationalen Beziehungen angesiedelter Forschungsgegenstand zunehmend aus der Untersuchungsperspektive der Vergleichenden Regierungslehre beleuchtet wird.

\section{Im Spannungsfeld: affirmative und kritische EU-Studien}

Zweifellos haben es die EU-Studien vermocht, sich innerhalb von etwa zwei Jahrzehnten $\mathrm{zu}$ einem bedeutenden und innovativen Forschungsbereich zu entwickeln. In wenigen anderen Bereichen der Sozialwissenschaft wird mit ähnlich hohem Methodenbewusstsein und einer solchen Vielfalt an Theorieansätzen zu einem aktuell relevanten Gegenstand gearbeitet. Auf diese Weise haben EU-Studien benachbarte Forschungsfelder befruchtet und dabei geholfen, bei Vertretern des ,area studies'-Ansatzes das Bewusstsein für methodische Standards zu verändern.

Handelt es sich bei EU-Studien jedoch um ein rein wissenschaftliches Anliegen empirischanalytischer Provenienz? Für einen guten Teil der EU-Forschung trifft dies sicher zu. Politische, rechtliche und wirtschaftliche Prozesse der europäischen Integration werden dementsprechend einer Fragestellung im sozialwissenschaftlichen Sinne unterzogen und im Weberschen Sinne wertneutral behandelt. Allerdings ist offenkundig, dass Teile der EU-Studien nicht diesem Leitbild folgen. Vielmehr befindet sich ein Teil der Integrationsforschung in einer finanziellen und organisatorischen Konfiguration, deren Prämissen auf eine EU-konforme, beziehungsweise affirmative, Grundhaltung hindeuten.

\section{Die Ambivalenz der europäischen Förderpolitik}

Zunächst betrifft der Vorwurf der Konformität jene Teile der EU-Forschung, die durch die Finanzierung durch EU-Institutionen gewissermaßen auf eine integrationsfreundliche Position geeicht sind. Hier stellt sich die Frage nach der Freiheit der Lehre mittlerweile ebenso scharf wie bei Forschungskooperationen zwischen Universitäten und Unternehmen. ${ }^{61}$ Zwar beeinträchtigt die europäisierte Forschungsförderung nicht unmittelbar die Freiheit einzelner Wissenschaftler. Dennoch geht vom europäischen Forschungsraum nach Ansicht einiger Beobachter die „Gefahr einer ,Lenkung“ des einzelnen Wissenschaftlers in Richtung bestimmter Forschungsrichtungen und -projekte“ aus. ${ }^{62}$ Gerade für jüngere Wissenschaftler oder finanzschwache Wissenschaftseinrichtungen kann beträchtlicher Druck entstehen, sich beispielsweise für das von der Europäischen Kommission finanzierte Programm zu qualifizieren, um beispielsweise im Rahmen des Jean-Monnet-Programms Finanzmittel für Lehrstühle, Exzellenzzentren, Kursmodule oder andere Informations- und Forschungsaktivitäten zu akquirieren. ${ }^{63}$

60 Simon Hix: The Study of the European Community: The Challenge to Comparative Politics, in: West European Politics 1/1994, S. 1-30, hier S. 2.

61 Siehe zum Beispiel Tanja Schultz: Deutsche Bank mischt bei Uni-Forschung mit, in: sueddeutsche.de, 30.5.2011.

62 Josef Franz Lindner: Die Europäisierung des Wissenschaftsrechts, in: Christian Flämig/Reinhard Grunwald/ Jürgen Heß u.a. (Hrsg.): Wissenschaftsrecht, Tübingen 2009, S. 86.

63 Europäische Kommission: Das Programm Jean Monnet zum Verständnis der europäischen Integration, abrufbar unter: http://ec.europa.eu/education/lifelong-learning-programme/doc88_de.htm (letzter Zugriff: 19.12.2011). 
Ein anderer Bereich, in dem die Europäische Union affirmative Wissensgenerierung und Wissensvermittlung begünstigt oder wenigstens begünstigen kann, besteht in der sozialwissenschaftlichen Forschungsförderung. Seit der Lissabon-Strategie des Jahres 2000, die Europa innerhalb eines Jahrzehnts zum ,wettbewerbsfähigsten und dynamischsten wissensbasierten Wirtschaftsraum der Welt" erheben soll, gehört die Förderung der Wissenschaft zu einem Kernanliegen der Europäischen Union. ${ }^{64}$ Während die EU-Förderung in vielen Forschungsfeldern als begrüßenswerte zusätzliche Finanzquelle gesehen werden kann und sollte, ergeben sich im Feld der Sozial-, Wirtschafts- und Geisteswissenschaften durchaus ambivalente Konsequenzen. Im Beschluss über das „7. Forschungsrahmenprogramm“ aus dem Jahr 2006 werden die möglichen Forschungsthemen in auffälliger Weise so gewählt, dass sie mit der Agenda der weiteren Vertiefung der Europäischen Union in Übereinklang stehen. Unterstützenswert sind EU-Studien, die unter anderem folgende Maßnahmen befördern:

„Entstehung eines demokratischen Mitverantwortungsgefühls und der aktiven Mitwirkung der Menschen in Europa; effektives und demokratisches staatliches Handeln auf allen Ebenen [...], um die Bürgerbeteiligung und Zusammenarbeit der öffentlichen und privaten Akteure zu verstärken; Forschungsarbeiten zur Entwicklung eines gemeinsamen Verständnisses und einer gemeinsamen Wertschätzung der europäischen Vielfalt und der Gemeinsamkeiten in den Bereichen Kultur, Religion, kulturelles Erbe, Institutionen und Rechtssysteme, Geschichte, Sprachen und Werte als Bausteine für unsere multikulturelle europäische Identität und unser multikulturelles europäisches Erbe. “65

In einer etwas anders gelagerten Form zeigen sich affirmative Tendenzen bei der Förderung von Lehrprogrammen. Diese bestehen zum Beispiel hinsichtlich der Abstimmung von Lehrangeboten, die für Studierende und sonstige Lernende einen Wechsel von Bildungssystemen ermöglichen sollen. In der Europawissenschaft gibt es verschiedene Initiativen zur Angleichung von Lehrprogrammen. Ein Beispiel ist das Projekt „Enhancing Political Science Teaching Quality and Mobility in Europe“, das von 2001 bis 2002 durchgeführt und von der Generaldirektion für Bildung und Kultur gefördert wurde. ${ }^{66} \mathrm{Im}$ Rahmen dieses Programms wurde die Einführung eines ,core curriculum‘ zur Angleichung der EU-Studien gefordert. Es sollten multinationale Lehrmodule eingerichtet werden, um die Mobilität von Studenten und Lehrenden zu unterstützen. Aus dem deutschsprachigen Raum kamen hierzu konkrete Reformvorschläge, ${ }^{67}$ welche zum Beispiel anhand der Ausgestaltung der Lehre sowie der Vorbereitung auf den Arbeitsmarkt konkretisiert wurden. ${ }^{68}$ Die Zielsetzung eines konvergenten Lehrplanes wurde mit der stetig wachsenden Komplexität der Union sowie dem Umfang von Ansätzen und Methoden zu deren Untersuchung begründet. Ein Aufgreifen von Anknüpfungspunkten zwischen Forschung und Lehre sowie die Zusammenführung europawissenschaftlicher Wissensbestände soll zur Kostenreduktion, Mobilität sowie einer

64 Europäischer Rat (Lissabon): Schlussfolgerungen des Vorsitzes, 23. und 24. März 2000, SN 100/00.

65 Siehe Beschluss Nr. 1982/2006/EG des Europäischen Parlaments und des Rates vom 18. Dezember 2006 über das Siebte Rahmenprogramm der Europäischen Gemeinschaft für Forschung, technologische Entwicklung und Demonstration (2007 bis 2013), in: Amtsblatt der EU, Nr. L 412 vom 30. Dezember 2012, S. 1-41, hier S. 25.

66 Ian Bache: The Europeanization of Higher Education: Markets, Politics or Learning?, in: Journal of Common Market Studies 2/2006, S. 231-248, hier S. 240.

67 Vergleiche Wolfgang Wessels/Ingo Linsenmann/Susanne Hägele: Teaching European Integration. A Core Curriculum on European Integration Studies, Paper presented at the ECSA Seventh Biennial International conference in Madison, Wisconsin, May 31-June 2, 2001.

68 Siehe Gaby Umbach/Bruno Scholl: Towards a core curriculum in EU studies, in: European Political Science 2/2003, S. 71-80. 
klareren Verortung innerhalb der Disziplinen beitragen. ${ }^{69}$ Allerdings ernteten die Befürworter eines ,core curriculum ' auch Widerspruch. ${ }^{70}$ So verwiesen Chris Rumford und Philomena Murray auf die Gefahr einer Zementierung dominanter Diskurse: „this is a recipe for a stultifying debate, boredom and sameness [...] as imposed conformity would result in a intellectual desert". 71

Die hier genannten Maßnahmen der EU-geförderten Forschung und Lehre sind nicht nur wegen der Tendenz zu Vereinheitlichung und Konformität ambivalent zu beurteilen. Vor allem erscheint problematisch, dass mit den genannten Förderprogrammen zwei Ziele vereint werden sollen, die nicht immer gleichzeitig zu erreichen sind. Einerseits geht es um auf Werturteilen aufbauendes, dann aber wertneutrales analytisches Wissen um die Zusammenhänge der europäischen Integration. Angesichts der Unterfinanzierung des universitären Sektors in vielen Mitgliedstaaten ${ }^{72}$ handelt es sich bei den EU-Mitteln nicht selten um Ressourcen, die qualitativ hochstehende EU-Forschung überhaupt erst ermöglichen. ${ }^{73}$ Andererseits stehen bei Projekten, die durch die Europäische Union explizit gefördert werden, politische Ziele im Sinne der Kommission als Ressourcen gewährender Behörde im Raum. Von der Europäischen Union geförderte Forschung hat häufig einen positiven Bezug auf eine Gesellschaftsordnung, in der ein vereintes Europa im Sinne der ,immer engeren Union der Völker Europas“74 im Mittelpunkt steht. Viele Europawissenschaftler dürften auch mit der Idee und Praxis von Erasmus-Intensivprogrammen vertraut sein, bei deren Beantragung und Bewilligung Partneruniversitäten aus vermeintlich benachteiligten Hochschulsystemen, etwa aus den osteuropäischen EU-Mitgliedstaaten, im Zweifelsfall relevanter sind als die Qualität der Lehrprogramme selbst.

Über lange Jahre wurde das affirmative und bisweilen symbiotische Verhältnis zwischen europäischen Institutionen in Form der Kommission und der begleitenden EU-Forschung nicht als problematisch gesehen. Die immer weiter gehende europäische Integration stellte für eine Mehrheit der Forscher ein unzweideutig wünschenswertes politisches Projekt dar, bei welcher die Entwicklung einer gemeinsamen europäischen Identität ebenso wie die weitere Vertiefung des EU-Institutionensystems als notwendig angesehen wurde. Wichtige EUForscher wie etwa Werner Weidenfeld fungierten beziehungsweise fungieren zugleich als Wissensgenerierer und politische Impulsgeber. Es war sicherlich auch kein Zufall, dass zu den Gründern des bereits erwähnten Arbeitskreises Europäische Integration Walter Hallstein sowie Alfred Müller-Armack gehörten. ${ }^{75}$ Politische und wissenschaftliche Akteure waren sich gewissermaßen einig, mit der europäischen Integration ein historisches Vermächtnis anzunehmen und die wissenschaftliche Auseinandersetzung ebenso wie die Lehr- und Forschungskooperation unter dem Leitbild einer guten Sache zu verfolgen.

\section{Kritische Europawissenschaft auf dem Vormarsch}

In jüngeren Jahren hat sich - insbesondere durch die Verträge von Maastricht und Lissabon - die ursprüngliche Konstellation geändert, in der sich die europäische Integration gewis-

69 Siehe Wessels/Linsenmann/Hägele: Teaching European Integration, 2001.

70 Als direkte Replik auf Umbach und Scholz zum Beispiel Chris Rumford/Philomena Murray: Do we need a core curriculum in European Union studies?, in: European Political Science 1/2003, S. 85-92.

71 Rumford/Murray: Do we need a core curriculum in European Union studies?, 2003, S. 85.

72 Organisation for Economic Co-operation and Development: Bildung auf einen Blick, Paris 2011, S. 271-308.

73 Bache: The Europeanization of Higher Education, 2006, S. 246.

74 Präambel des Vertrags über die Europäische Union.

75 Siehe Arbeitskreis Europäische Integration: Willkommen beim AEI, abrufbar unter: http://www.aei-ecsa.de/ (letzter Zugriff: 21.2.2012). 
sermaßen im politikfreien Raum vollzog. Im Verhältnis zu den nationalen Demokratien entwickelt sich die europäische Institutionenordnung invasiv, indem einerseits nationale Handlungsspielräume beschnitten und andererseits Angelegenheiten der europäischen Politik zum Gegenstand von traditionell innenpolitischen Diskussionen werden. ,Europa' kann konsequenterweise nicht mehr allein als „Rettungsanker"76 vor dem Nationalen dienen. Politische Angelegenheiten jenseits der Vertiefungsintegration entwickeln sich zu Streitfällen, in denen europäische Akteure und Institutionen sowie die EU-Politik im Ganzen zum Gegenstand wissenschaftlicher Kritik werden oder werden können.

Im Windschatten dieses Prozesses entwickelten sich Teile der sozialwissenschaftlichen EU-Studien zu einer kritischen Europawissenschaft. Nicht nur einzelne Aspekte der europäischen Integration, sondern die Fundamente des Einigungswerks werden zum Teil vehement infrage gestellt. Vor etwa 15 Jahren beließ es Peter Graf Kielmansegg bei - seinerzeit bereits deutlichen - Hinweisen auf die schwache Demokratiefähigkeit der Europäischen Union. ${ }^{77}$ Wenige Jahre später ist daraus eine umfassende Anklage gegen die politischen Eliten Europas geworden, die sich trotz eines mangelnden gesellschaftlichen Fundaments zu einem politischen System entschlossen haben, das aus der Sicht mancher wissenschaftlicher Beobachter schwerwiegende Defizite aufweist. ${ }^{78}$ Die These, zwischen bürokratischer Technokratie und Demokratie existiere ein Gegensatz, ist von der politischen Essayistik, wie etwa bei Hans Magnus Enzensberger, ${ }^{79}$ in die politikwissenschaftliche Auseinandersetzung gewandert.

Ein Beispiel für die neue wissenschaftliche EU-Kritik bildet die Debatte über die gegenwärtige und künftige Institutionenordnung der Europäischen Union. Vor einigen Jahren wurden mögliche problematische Aspekte des fusionierten Institutionensystems wohlwollend und nicht mit alarmistischen Untertönen diskutiert. ${ }^{80}$ Wenig später sprach Hans Herbert von Arnim - an der Verwaltungshochschule Speyer ${ }^{81}$ in einer für die Beamtenweiterbildung zentralen Position - vom „Europa-Komplott““. Der Untertitel des Buches lautet: „wie EUFunktionäre unsere Demokratie verscherbeln“" ${ }^{82}$ Neben dem konservativen Feuilleton wurde die Publikation auch seitens der Wissenschaft durchaus wohlwollend aufgenommen. ${ }^{83}$ Die harten Töne von Arnims können nicht mehr als Einzelfall bezeichnet werden. Harsche wis-

76 Wilfried Loth: Rettungsanker Europa? Deutsche Europa-Konzeptionen vom Dritten Reich bis zur Bundesrepublik, in: Hans-Erich Volkmann (Hrsg.): Ende des Dritten Reiches - Ende des Zweiten Weltkrieges. Eine perspektivische Rückschau, München 1985, S. 201-221.

77 Peter Graf Kielmansegg: Integration und Demokratie, in: Markus Jachtenfuchs/Beate Kohler-Koch (Hrsg.): Europäische Integration, Opladen 1996, S. 47-72.

78 Max Haller: European Integration as an Elite Process. The Failure of a Dream? London/New York 2008; Jürgen Neyer: Die halbierte Gerechtigkeit in der Europäischen Union, in: Leviathan 1/2007, S. 30-46.

79 Hans Magnus Enzensberger: Ach Europa! Wahrnehmungen aus sieben Ländern mit einem Epilog aus dem Jahre 2006, Frankfurt Main 1987; Hans Magnus Enzensberger: Sanftes Monster Brüssel oder die Entmündigung Europas, Frankfurt Main 2011.

80 Vgl. Dieter Grimm: Vertrag oder Verfassung? Die Rechtsgrundlage der Europäischen Union im Reformprozess Maastricht II, in: Dieter Grimm/Joachim Jens Hesse/Reimut Jochimsen u.a. (Hrsg.): Zur Neuordnung der Europäischen Union: Die Regierungskonferenz 1996/1997, Baden-Baden 1997, S. 9-31.

81 Heute Deutsche Universität für Verwaltungswissenschaften Speyer.

82 Hans Herbert von Arnim: Das Europa-Komplott. Wie EU-Funktionäre unsere Demokratie verscherbeln, München 2006.

83 Als Auswahl: Jürgen Elvert: Viel zu wenig Kontrolle, in: Frankfurter Allgemeine Zeitung, 28.2.2007; Andreas Eis, in: Zeitschrift für Politikwissenschaft 3/2006, S. 1124; Thorsten Moeck: „Abrechnung mit korrupten Eurokraten“, in: Kölner Stadtanzeiger, 10.3.2006; Rudolf Wassermann: Die europäische Pseudodemokratie, in: Recht und Politik. Vierteljahreshefte für Rechts- und Verwaltungspolitik 3/2006, S. 185-186; Holger Eichele: Krake außer Kontrolle, in: Münchner Merkur, 10.3.2006; Ralf Joas: Undemokratisch, bürgerfern, maßlos, in: Die Rheinpfalz, 28.9.2006. 
senschaftliche Kritik wurde auch am Europäischen Gerichtshof geübt; nach Martin Höpner vom Max-Planck-Institut für Gesellschaftsforschung sei dieser als „Usurpator“ der europäischen Politik zu sehen. ${ }^{84}$

Verschärft wird der kritische Unterton durch die europäische Schulden- und Finanzkrise. So äußerten sich bereits in den 1990er Jahren deutsche Wirtschaftswissenschaftler kritisch zur Einführung des Euro. ${ }^{85}$ Seinerzeit handelte es sich um eine isolierte Position, die letztlich mit den Wurzeln der Volkswirtschaftslehre - der Nationalökonomie - erklärt werden konnte. Im Zuge der Krise werden die Unzulänglichkeiten des europäischen Währungssystems indes in einen viel weiteren Zusammenhang gebracht. Der politische Umgang mit der Krise wird vor dem Szenario des „Schuldensozialismus“ (Hans-Werner Sinn) diskutiert. ${ }^{86}$ Für Fritz W. Scharpf gehört die Eurokrise in den Kontext der fahrlässig und selbstverschuldet geschwächten Steuerungsfähigkeit der Politik. Die nicht verwirklichte Wirtschafts- und Währungsunion ist nach seiner Ansicht ein Indiz dafür, dass die politischen Eliten in Europa sich von der traditionellen Funktion der Abschwächung von Marktrisiken weitgehend verabschiedet haben. ${ }^{87}$ Noch grundsätzlicher stellt Wolfgang Streeck die Frage, ob die Europäische Union sowie die europäische Politik in der Lage seien, das kapitalistische System in sozial und demokratisch verträglicher Weise einzubinden. ${ }^{88}$

Hieraus wird ersichtlich, dass sich mittlerweile wichtige Vertreter der sozialwissenschaftlichen EU-Studien von einer EU-affirmativen Grundhaltung abgewendet haben und mitunter fundamentale Kritik am europäischen Herrschaftssystem formulieren. Gewisse Parallelen lassen sich zur bundesdeutschen Politikwissenschaft der 1960er und 1970er Jahre ziehen. Diese war als Demokratiewissenschaft entstanden und vertrat in den Gründungsjahren das Ziel, der deutschen Bevölkerung durch Wissens- und Wertevermittlung das demokratische System näher zu bringen. Erst als das Bewusstsein für - reale oder vermeintliche - Missstände des demokratischen Systems geschärft war, kamen die kritischen Sozialwissenschaften auf. ${ }^{89}$ Die Konsequenzen für die EU-Studien liegen auf der Hand. Da sich die Politikwissenschaft (auch) als Demokratiewissenschaft versteht, werden (reale oder vermeintliche) Defizite der europäischen Demokratie zunehmend schärfer problematisiert. Entsprechend haben sich viele Vertreter der Europawissenschaft entschieden, die EU-Institutionen als das anzusehen, was in vielen Positionspapieren jahrzehntelang gefordert wurde - als politisch bedeutsame Herrschaftsträger, denen gegenüber kritische Distanz ein wichtiges Kriterium für unabhängige Wissenschaft darstellt.

84 Martin Höpner: Usurpation statt Delegation. Wie der EuGH die Binnenmarktintegration radikalisiert und warum er politischer Kontrolle bedarf, in: Max-Planck-Institut für Gesellschaftsforschung: MPIfG Discussion Paper 12/2008; ähnlich auch Fritz W. Scharpf: Legitimität im europäischen Mehrebenensystem, in: Leviathan 2/2009, S. $244-280$

85 Am pronociertesten Wilhelm Hankel/Wilhelm Nölling/Karl Albrecht Schachtschneider u.a.: Die Euro-Klage. Warum die Währungsunion scheitern muss, Reinbek 1998.

86 Hans Werner Sinn: Kein Schuldensozialismus!, in: Handelsblatt, 6.1.2012.

87 Fritz W. Scharpf: Die Eurokrise: Ursachen und Folgerungen, in: Zeitschrift für Staats- und Europawissenschaften 3/2011, S. 324-337.

88 Wolfgang Streeck: Demokratischer Kapitalismus und europäische Integration. Ein Epilog, in: Lettre International 95/2011, S. 11-14; Wolfgang Streeck: Die Krisen des demokratischen Kapitalismus. Inflation, staatliche Defizite, private Verschuldung, faule Kredite, in: Lettre International 95/2011, S. 6-10.

89 Jürgen W. Falter/Felix W. Wurm: Politikwissenschaft in der Bundesrepublik Deutschland, Opladen 2003. 